\title{
Estudio isotérmico de biosorción de plomo en aguas utilizando residuos vegetales
}

\author{
Christian Larenas Uría*, Andrango Daniel, Inga Pablo
}

Centro de Investigación para la Valoración de la Biodiversidad (CIVABI), Universidad Politécnica Salesiana. Quito, Ecuador.

* Autor para correspondencia: clarenas@ups.edu.ec

\begin{abstract}
Resumen
Los metales pesados presentes en aguas industriales de desecho constituyen un problema ambiental de gran magnitud en razón de su elevada toxicidad para los organismos vivos. La posibilidad de desarrollar métodos de remoción de estos contaminantes resulta particularmente atractiva al tomar como punto de partida el uso de materias primas de bajo costo, que de otra forma pueden ser consideradas como residuos sin utilidad. Dentro de esta categoría se hallan los desechos vegetales provenientes de actividades domésticas o agroindustriales. Bajo estos criterios se estudió la capacidad de biosorción de materiales celulósicos en función del $\mathrm{pH}$ y tiempo de biosorción, siendo el $\mathrm{pH}$ óptimo igual a 6,0 y determinándose la mayor tasa de sorción entre 15 y 30 minutos. Además mediante un estudio isotérmico se calculó la capacidad máxima del biosorbente $\left(q_{\text {máx }}\right)$, siendo esta en condiciones óptimas de $182 \mathrm{mg}$ plomo/g biosorbente. Todos los resultados se obtuvieron a $22^{\circ} \mathrm{C}$.
\end{abstract}

Palabras clave: Estudio Isotérmico, biosorción, contaminación por plomo.

\section{Introducción}

Existen diversas fuentes de contaminación por plomo de aguas naturales, siendo la principal la descarga de efluentes industriales directamente a fuentes naturales sin ningún tratamiento previo. Este problema se debe tanto a los altos costos como a la baja eficiencia de muchos de los métodos empleados para la remoción de los metales pesados. En la actualidad se ha desarrollado una diversa gama de tecnologías que apuntan a ser soluciones de bajo costo y ambientalmente amigables. Una de estas técnicas es la llamada biosorción, en la cual se hace uso de materia orgánica -viva, muerta o inmovilizada- para la remoción de metales pesados y su posterior recuperación (Sánchez et al. 2008).
El presente trabajo tiene por objeto evaluar la capacidad de biosorción, mediante un estudio isotérmico (Gutiérrez et al. 2008) de material procedente de residuos de jardines domésticos tomando en consideración criterios como el $\mathrm{pH}$ y el tiempo de contacto entre el biosorbente y el contaminante. Se espera que a través de un proceso controlado de hidrólisis, la estructura celulósica del material presente una gran cantidad de sitios activos para la adsorción o retención de iones $\mathrm{Pb}^{2+}$ en solución.

\section{Materiales y métodos}

\section{I Reactivos}

Se utilizaron únicamente reactivos de grado analítico. Las soluciones de $\mathrm{Pb}^{2+}$ fueron preparadas a partir de 


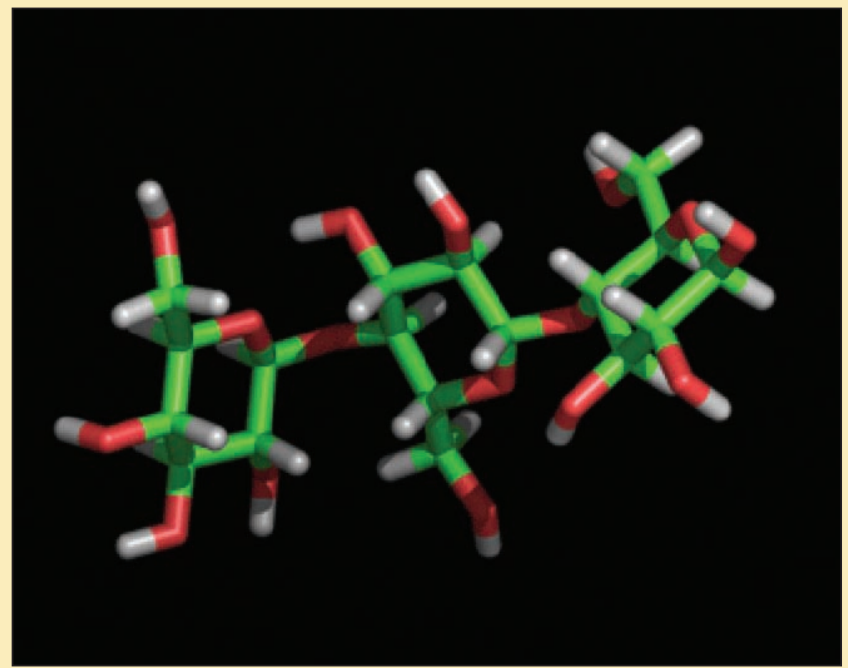

una solución estándar certificada de 1000 ppm de $\mathrm{Pb}^{2+}$. Para el ajuste del $\mathrm{pH}$ se prepararon soluciones buffer así como soluciones de $\mathrm{NaOH}$ y $\mathrm{HNO}_{3} 0, \mathrm{I} \mathrm{M}$.

\subsection{Obtención del Biosorbente}

Se utilizó una mezcla al azar de desechos de jardín doméstico que se sometió al secado durante 15 días. La muestra fue molida y tamizada, luego de lo cual se hidrolizó de forma ácida y alcalina mediante reflujo durante 4 horas. El material obtenido se desecó a $60^{\circ} \mathrm{C}$ durante 3 días.

\subsection{Efecto del pH}

En vasos de precipitación se colocó $50 \mathrm{ml}$ de solución de $1,0 \mathrm{ppm} \mathrm{Pb}^{2+}$. Se ajustó el $\mathrm{pH}$ de las soluciones a 3,$0 ; 4,0 ; 5,0 ; 6,0 ; 7,0$ y 8,0 y a cada una se agregó $0.1 \mathrm{~g}$ de biosorbente. Las soluciones se agitaron durante 2 horas luego de lo cual fueron filtradas, utilizándose la solución resultante para determinar la capacidad de biosorción de plomo en función del $\mathrm{pH}$.

\subsection{Cinéticas de Biosorción}

La cinética de biosorción se determinó colocando en vasos de precipitación $50 \mathrm{ml}$ soluciones de 50,70 , 100,500 y 1000 ppm de $\mathrm{Pb}^{2+}$. A cada solución se agregó $0, \mathrm{I} g$ de biosorbente y se ajustó a $\mathrm{pH}$ óptimo. Las soluciones se mantuvieron en agitación a 200 rpm durante I, 5, I0, 15, 30, 60, 90 y 120 minutos. Cumplidos los tiempos respectivos, las soluciones fueron filtradas y el resultante fue analizado para determinar la concentración del metal.

\subsection{Isotermas de Biosorción}

Para este estudio se consideró la utilización de los modelos isotérmicos de Langmuir y de Freundlich. En cada caso la metodología consistió en colo- car en vasos de precipitación $50 \mathrm{ml}$ de soluciones de $\mathrm{Pb}^{2+}$ de 50, 500, 700, 1000 y 2000 ppm. A cada solución se agregó $0, \mathrm{I} g$ de biosorbente, se ajustó a $\mathrm{pH}$ óptimo y se mantuvo en agitación durante 30 minutos. Posteriormente las soluciones fueron filtradas $y$ la solución resultante se utilizó para evaluar la capacidad máxima de retención $\left(\mathrm{q}_{\text {máx }}\right)$ de plomo.

Los cálculos se realizaron tomando en cuenta las siguientes relaciones:

$$
q=\frac{(\mathrm{Ci}-\mathrm{Ce}) * V}{X_{0}}
$$

Donde:

$q$ = Tasa de biosorción de $\mathrm{Pb}^{2+}$ (mg/g de plomo por gramo de biosorbente)

$\mathrm{Ci}=$ Concentración inicial de $\mathrm{Pb}^{2+}$ en solución $(\mathrm{mg} / \mathrm{l})$

$\mathrm{Ce}=$ Concentración final de $\mathrm{Pb}^{2+}$ (en equilibrio) en solución $(\mathrm{mg} / \mathrm{l})$

$X_{0}=$ Cantidad de biosorbente agregada $(\mathrm{g})$

$V=$ Volumen de solución (I)

Para la isoterma de Langmuir se linealizó la ecuación graficando I/q vs. I/Ce, obteniéndose la siguiente relación:

$$
\frac{\mathrm{I}}{\mathrm{q}}=\frac{\mathrm{l}}{\mathrm{q}_{\text {máx }}}+\frac{\mathrm{l}}{\mathrm{b} * \mathrm{q}_{\text {máx }} * \mathrm{Ce}}
$$

Donde:

$q_{\text {máx }}=$ Tasa máxima de retención de plomo $(\mathrm{mg}$ de $\mathrm{Pb} / \mathrm{g}$ biosorbente)

$b=$ constante relacionada con la afinidad del biosorbente por los iones $\mathrm{Pb}^{2+}$

Pudiendo evaluarse la isoterma de Langmuir a partir de un factor adimensional $R_{L}$, así:

$$
R_{L}=\frac{1}{I+b * C i}
$$

Donde los valores de $R_{L}$ indican:

$R_{L}=0$ Sistema isotérmico irreversible.

$0<R_{L}<$ I Sistema isotérmico favorable, siendo $\mathrm{R}=\mathrm{I}$ lineal 
$R_{L}>$ I desfavorable, debido a que se observa que la sorción del metal no es significativa.

Para la isoterma de Freundlich la ecuación usada fue:

$$
\log q=\frac{I}{n} \log C e+\log K
$$

\section{Donde:}

$n=$ Constante relacionada con la intensidad de sorción del biosorbente en función de su grado de heterogeneidad.

$K=$ Constante de Freundlich relacionada a la capacidad de sorción del biosorbente.

\subsection{Análisis de las muestras}

El $\mathrm{Pb}$ contenido en todas las muestras se analizó mediante la técnica de Absorción Atómica utilizando un equipo Varian 20, trabajando a dos longitudes de onda. Para las soluciones de I,0 a 50,0 ppm se realizaron las mediciones a $283,3 \mathrm{~nm}$ y para las soluciones de 50 a 2000 ppm a 205,3 nm.

\section{Resultados}

\section{I Efecto del $\mathrm{pH}$}

De acuerdo a lo observado en la figura I, el pH óptimo para la biosorción de plomo es igual a 6,0. En los valores superiores e inferiores a este $\mathrm{pH}$ se aprecia una disminución progresiva de la biosorción; en valores superiores a $\mathrm{pH} 8$ el $\mathrm{Pb}^{2+}$ en solución empieza a precipitar como $\mathrm{Pb}(\mathrm{OH})_{2}$ y por tanto existe menos plomo disponible para ser retenido por el biosorbente. En valores de $\mathrm{pH}$ por debajo de 4 existe una competencia por los sitios activos del biosorbente entre los iones $\mathrm{Pb}^{2+}$ y los iones $\mathrm{H}^{+}$.

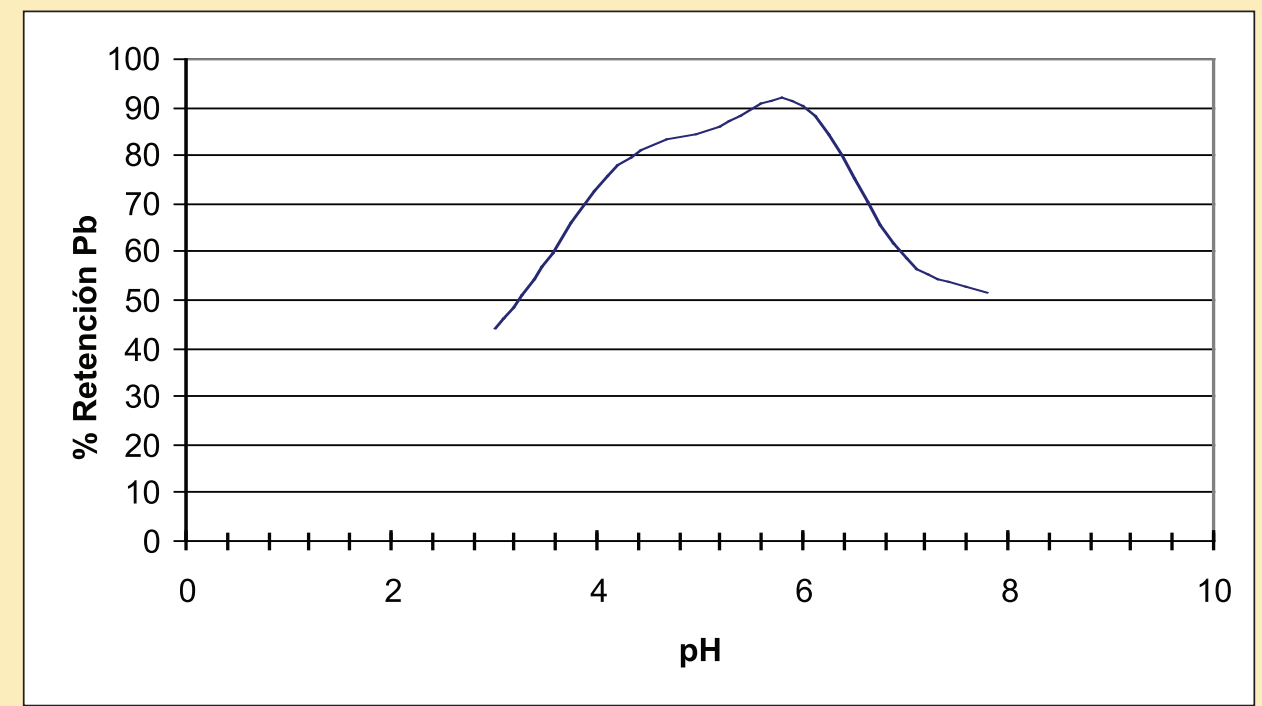

Figura I. Resultados experimentales acerca del efecto del $\mathrm{pH}$ sobre la Biosorción de $\mathrm{Pb}^{2+}$. 


\subsection{Cinéticas de Biosorción}

Los resultados obtenidos, mostrados en la figura 2 denotan que la máxima biosorción de $\mathrm{Pb}^{2+}$ se produce entre los 15 y 30 minutos; sin embargo, para soluciones más diluidas (hasta 100 ppm) el valor máximo de biosorción se produce a los 5 minutos. Esto representa una saturación progresiva del biosorbente, es decir, que el $\mathrm{Pb}^{2+}$ se une más débilmente a la superficie del biosorbente a medida que éste se va saturando.

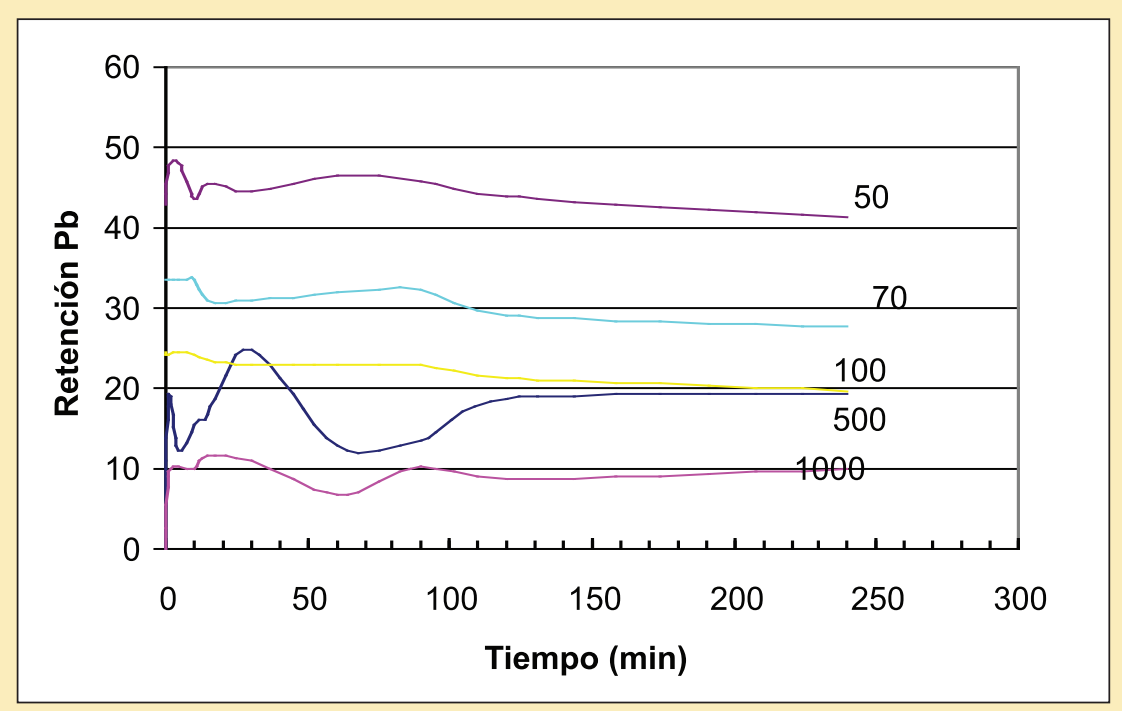

Figura 2. Cinética de Biosorción de $\mathrm{Pb}^{2+}$. Los tiempos ideales de biosorción son de 15 a 30 min para bajas concentraciones y de 5 min para altas concentraciones.

\subsection{Isotermas de Biosorción}

Los resultados, de acuerdo al modelo de Langmuir presentados en la figura 3 , nos permiten calcular la ordenada al origen $1 / q_{\text {máx }}$ y la pendien- te $1 / b^{*} q_{\text {máx }}$ con lo cual $q_{\text {máx }}$ resultante es igual a $182 \mathrm{mg}$ de $\mathrm{Pb} / \mathrm{g}$ biosorbente.

Utilizando la ecuación (3), el análisis de los valores de $R_{L}$ (Tabla I) denota un comportamiento isotérmico favorable $\left(0<R_{L}<1\right)$.

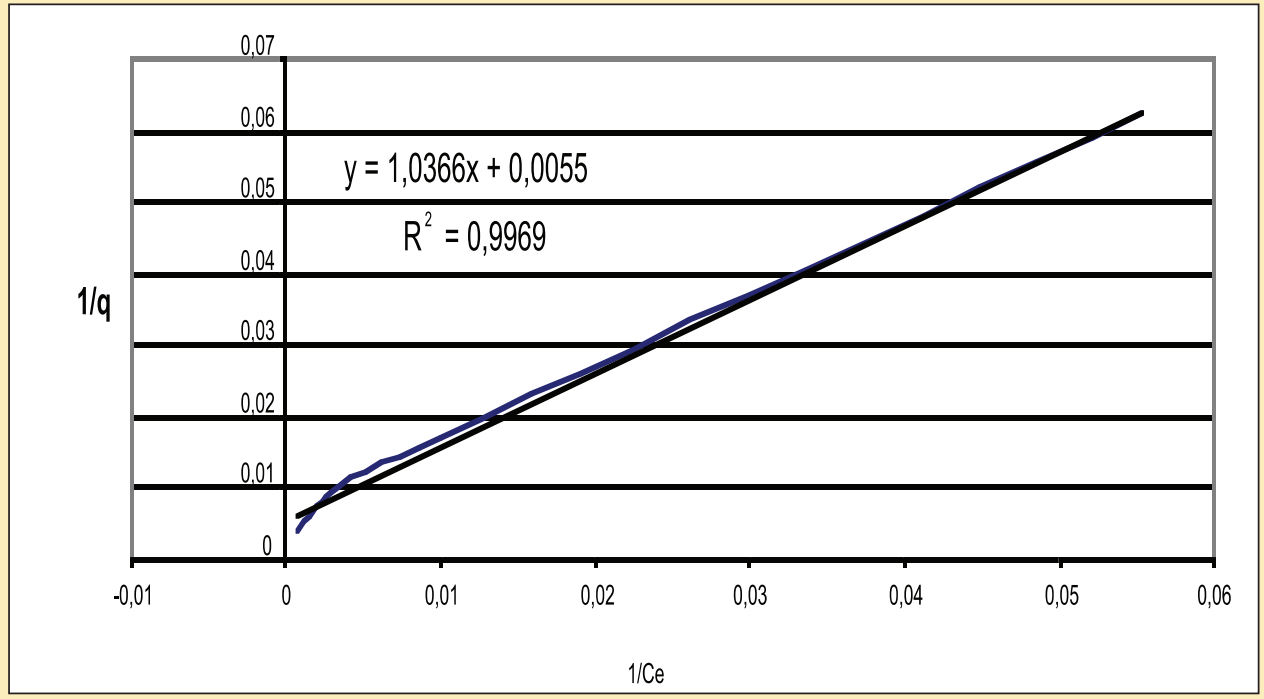

Figura 3. Isoterma de Langmuir, se aprecia que los datos se ajustan en un $99,69 \%$ al modelo propuesto. 
Tabla I. Resultados de biosorción de $\mathrm{Pb}^{2+}$, se aprecia claramente como los valores de $\mathrm{R}$ se mantienen entre 0 y I.

\begin{tabular}{llll}
\hline$C i[\mathrm{ppm}]$ & $C e[\mathrm{ppm}]$ & $Q[\mathrm{mg} / \mathrm{g}]$ & $R_{L}$ \\
\hline 1 & 0,1 & 0,09641807 & 0,9947222 \\
5 & 0,3 & 0,28894775 & 0,97415656 \\
10 & 1,2 & 1,15030675 & 0,94961524 \\
50 & 18,1 & 15,930995 & 0,79033242 \\
500 & 313 & 113,48392 & 0,27375482 \\
700 & 458 & 128,81089 & 0,21213113 \\
1000 & 702 & 143,335511 & 0,15858397 \\
2000 & 1528 & 161,854119 & 0,08612067 \\
\hline
\end{tabular}

Para el modelo de Freundlich la ecuación (4) se presenta (OFigura 4) permitiendo calcular $K=2,76$ y $n=1,64$, demostrándose, por tanto, una afinidad significativa del biosorbente por el $\mathrm{Pb}^{2+}(n>\mathrm{I})$ así como una fuerza de adsorción intensa del metal por la matriz de adsorción.
Ambos modelos presentan coeficientes de correlación $\left(\mathrm{R}^{2}\right)$ muy cercanos a la unidad, así el comportamiento isotérmico de sorción del biosorbente puede evaluarse adecuadamente por cualquiera de ellos.

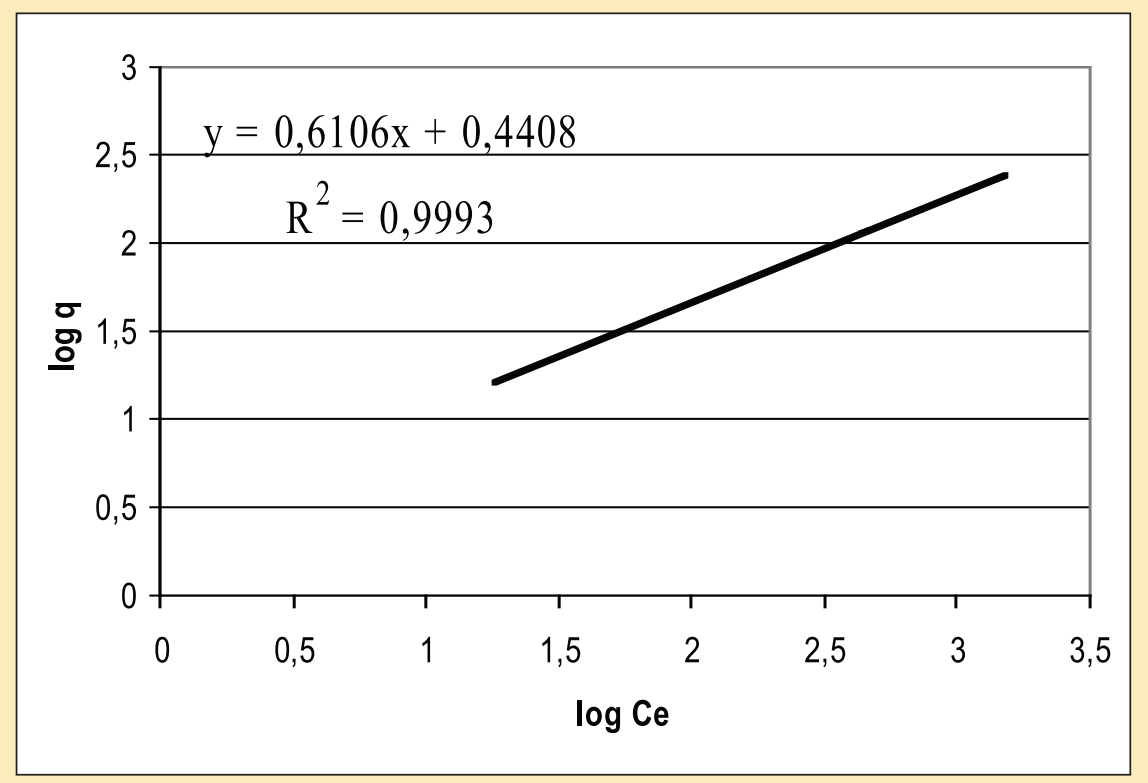

Figura 4 Isoterma de Freundlich, los resultados obtenidos se ajustan en un 99.93\% a la isoterma propuesta.

\section{Discusión}

El biosorbente desarrollado presenta un comportamiento óptimo para la sorción del $\mathrm{Pb}^{2+}$ cuando el $\mathrm{pH}$ de la solución es igual a 6,0; sin embargo, con valores cercanos hasta $\mathrm{pH}=5$ la tasa de retención del metal es todavía significativa.

La cinética de biosorción indica que para soluciones de hasta 100 ppm de $\mathrm{Pb}^{2+}$ la cantidad máxi- ma retenida de metal se produce a los cinco minutos desde que se pone en contacto al biosorbente con la solución; en concentraciones superiores a 500 ppm el tiempo óptimo está comprendido entre 15 y 30 minutos.

Tanto el modelo de Langmuir como el de Freundlich se ajustan idealmente para describir las isotermas de biosorción del sistema $\left(R^{2}=0,99\right)$. Sin embargo el sistema de Langmuir linealizado en función de $\mathrm{Ce}$ 


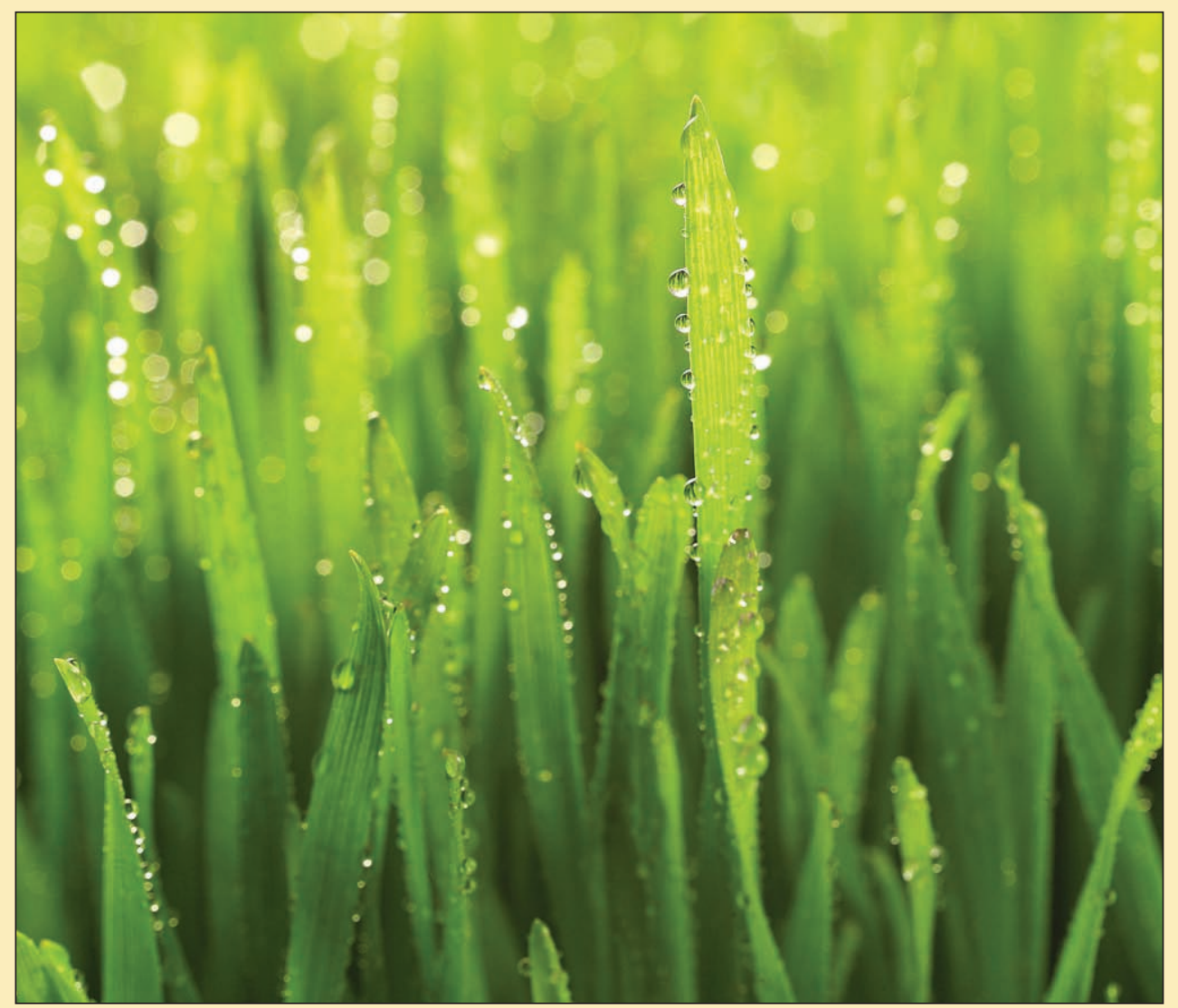

presenta menor linealidad $\left(R^{2}=0,90\right)$ pero un mayor valor de $q_{\text {máx }}\left(q_{\text {máx }}=333 \mathrm{mg} \mathrm{Pb} / \mathrm{g}\right)$. Por otra parte al obtener las ecuaciones de regresión tomando como referencia datos exclusivamente de alta concentración, a partir de 500 ppm, $q_{\text {máx }}$ aumenta hasta un valor de $360 / \mathrm{mg}$ de $\mathrm{Pb} / \mathrm{g}$ biosorbente.

Este método de biosorción es altamente recomendado, por lo que es importante plantear su estudio con otros metales pesados.

\section{Agradecimiento}

Los autores agradecen a la Universidad Politécnica Salesiana por el financiamiento a este trabajo, a través del proyecto "Obtención de un biosorbente por modificación química o física de residuos vegetales", así como a todas las personas que directa o indirectamente contribuyeron a abrir los espacios físicos, espirituales y mentales para la realización del mismo.

\section{Referencias}

GUTIÉRREZ, M., GONZÁLEZ, L, SÁNCHEZ, E., MELLADO, D. 2008. Biosorcion de $\mathbf{P b}^{2+}$ por biomasa de Saccharomyces cerevisiae. Federación Mexicana de Ingeniería Sanitaria e Ingenierías Ambientales. En línea:

$<$ http://www.femisca.org/publicaciones/XIcongreso/XICNIS089.pdf>

SÁNCHEZ, E., GONZÁLEZ, G., ALMAGUER, CANTÚ,V., SÁENZ T., LIÑÁN, I., Estudio cinético e isotermas de adsorción de Ni (II) y Zn (II) utilizando biomasa del alga Chlorella sp. Inmovilizada. En línea: <http://dialnet.unirioja.es/servlet/articulo?codigo $=2565693>$

SAIFUDDIN, N.; RAZIAH, A.Z.: Remoal of Heavy Metals from Industrial Effluent Using Saccharomyces Cerevisiae (Baker's Yeast) Immobilised in Chitosan/lignosulphonate Matrix. En línea:

<http://www.insinet.net/jasr/2007/209 I-2099.pdf> 